\title{
FEASIBILITY OF PLASMA TECHNOLOGY UTILIZATION IN CONCRETE INDUSTRY
}

\author{
Kasra Shafiei $^{1}$, Bernd Hillemeier ${ }^{2}$ \\ ${ }^{I}$ Department of Building Materials and Construction Chemistry, Institute Civil Engineering, Technische Universität \\ Berlin, Berlin, Germany \\ ${ }^{2}$ Department of Building Materials and Construction Chemistry, Institute Civil Engineering, Technische Universität \\ Berlin, Berlin, Germany
}

\begin{abstract}
Concrete technology is growing in different directions as various sciences are feeding this industry regarding the huge demand for concrete each day all around the world. In spite of this fact there is a lack of research in the field of plasma technology and its possible usages in the concrete. Plasma technology as a relatively recent achievement of mankind has many applications in different industries and one of its main utilizations is the surface modification of materials and specially polymers with changing the wettability and adhesion properties.

In this research, the main endeavor is to study the possibility of plasma surface modification in concrete mainly within 2 concepts; first to enhance the adhesion of cement paste to the aggregates to improve the interfacial transition zone and thus improving the concrete properties such as compressive strength and impermeability. This concept is followed by measuring the compressive strength and porosity of the concrete samples, which are made with and without plasma treatment. The second concept is to change the wettability of the release agent materials on the surface of the mold to enhance the final surface of the concrete after demolding. This part is done by comparing the visual observations of fresh cement slurry and hardened cement paste on the surface of different release agents before and after treating with plasma.
\end{abstract}

Keywords: Plasma, Corona Discharge Treatment, Cement Matrix, Adhesion, Wettability, Release Agent, Concrete Final Surface, Compressive Strength, Porosity.

\section{INTRODUCTION}

In the recent decades, we have witnessed the application of different methods and techniques to improve the concrete properties, decrease the production costs and minimize the environmental pollutions concerning cement and concrete productions. One of these methods is to modify the surface properties of concrete materials such as aggregates in order to improve mostly all of the concrete features. In other words, modifying surface features of concrete elements and mainly the aggregates could result in improvement of concrete properties in general.

In spite of the wide range of research projects so far, our knowledge is limited about the effect of plasma technology on cement and concrete behavior. In fact, it is not clear how it is possible to use benefits of plasma technology and influence the properties of concrete with it. Plasma, a popular technique in industry at the moment, is one of the most promising methods to affect the properties of different surfaces diversely and effectively.

This research paper is an attempt to introduce and highlight two possible ways to apply plasma technology and its challenges in cement and concrete industries.

\subsection{Plasma and Corona Discharge}

The term plasma is often referred to as the fourth state of matter. As temperature increases, molecules become more energetic and transform in the sequence: solid, liquid, gas, and plasma. In the latter stages, molecules in the gas dissociate to form a gas of atoms and then a gas of freely moving charged particles, electrons, and positive ions [1].

In another word, plasma is similar to gas in which a certain portion of the particles are ionized. Heating a gas may ionize its molecules or atoms, thus turning it into plasma which contains charged particles: positive ions and negative electrons or ions [2]. Plasma is by far the most common phase of ordinary matter in the universe, both by mass and by volume [3].

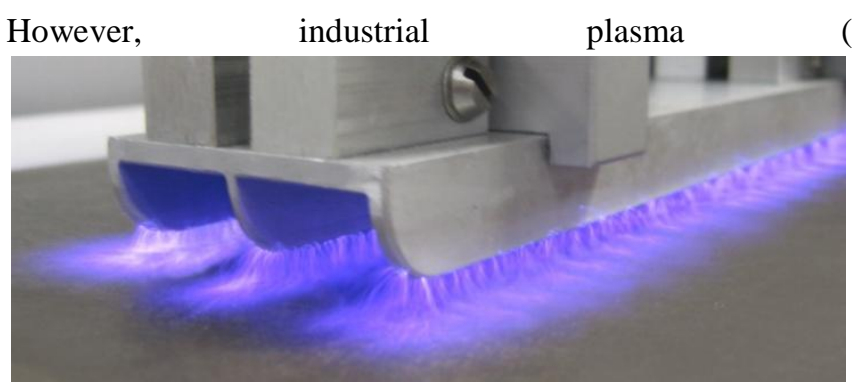


Fig 1), a relatively new achievement comparing to concrete, is growing in application and becoming more popular every day due to its unique capabilities.

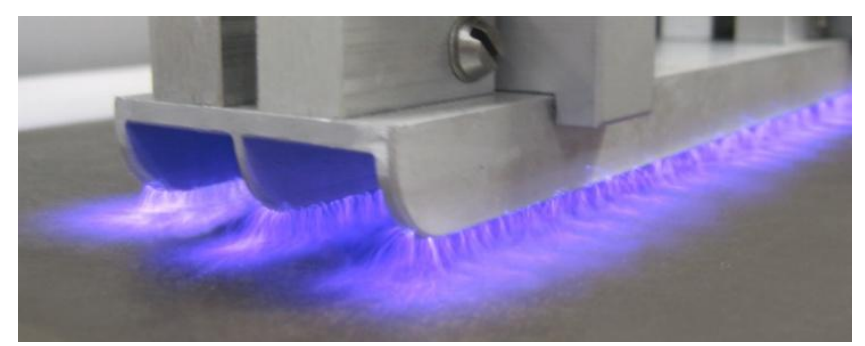

Fig 1 [4]: A units of plasma treatment

The plasma is used widely both in scientific studies and in different industries such as food and agriculture, textile, packaging industries, medicine, etc. Plasma itself is available in many different types owing to numerous parameters such as kind of generating pole, power, availability and flow of different types of gas during the treatment, temperature, treatment duration, etc.

One of the special capabilities of plasma treatment is the possibility of using different types of gas during the treatment of any substrate. This results in diverse achievements, one of which is to settle a layer of the applied gas on the treated substrate; in another word, coating the substrate.

However, one of the main applications of plasma in common industries is surface modification of the materials. By means of plasma surface modification, desired surface characteristics (mainly in polymeric based materials) can easily be achieved without changing the bulk properties of substrates [5]. An example for effects of plasma surface treatment in changing the wettability of a steel plate is presented in Figure2.
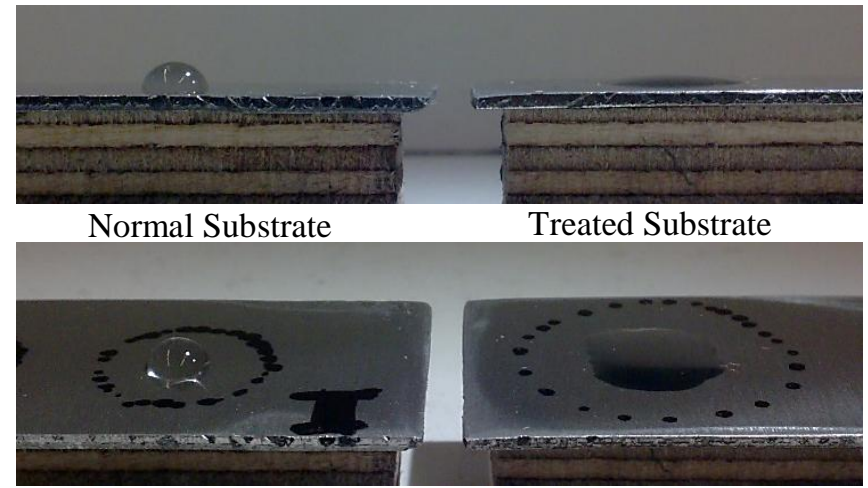

Fig 2: Showing the effect of plasma treatment on the wettability of a steel substrate

Corona treatment, a similar process in several respects, has been in industrial use for several decades. However, unlike corona, in which the reactive gas is usually air and which is generally restricted to simple surface geometries such as flexible webs, plasma treatment is extremely versatile in its capabilities [6].
The mechanism of changing the surface properties with plasma or corona treatment is not always the same. It depends mainly on the material type of the treated element and also on the type of treatment itself. However, there are four mechanisms due to which the surface modification takes place:

1- Cleaning effect [6]: making the surface clean of organic contamination.

2- Crosslinking or branching (chemical influence) of near-surface molecules which can cohesively strengthen the surface layer $[6,7]$.

3- Modification of surface-chemical structure (chemical influence), which can occur during plasma treatment itself, and upon re-exposure of the treated part to air, at which time residual free radicals can react with atmospheric oxygen or water vapor [6]. A SEM picture of PET fibers in Fig 3 shows the formation (grafting) on the surface due to plasma treatment.

4- Ablation or etching (physical influence) [6], which can remove a weak boundary layer and increase the surface area.
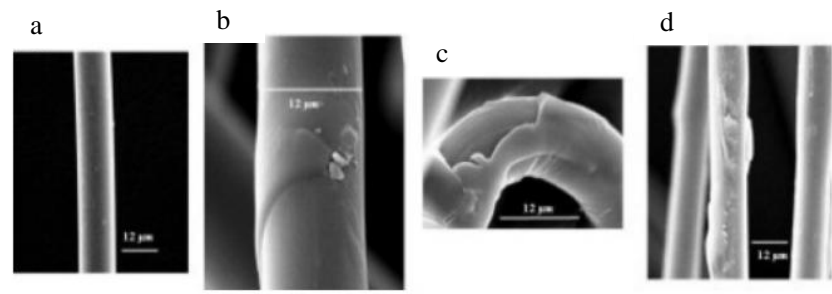

Fig 3 [8]: Illustration of the treatment physical effect. SEM image of Poly Ethylene Terephthalate (PET) Fibers with $12 \mu \mathrm{m}$ thickness; native (a) and grafted after two cycles of plasma treatment (b, c, and d)

Although plasma is applicable in many industries, its application and benefits are poorly known to the civil engineering sector. The only research subject which encompasses the both topics of plasma and concrete is the application of plasma in order to increase the bond forces between the cement matrix and polymeric fibers. In these groups of researches [5-9] different plasma techniques are used on polymeric fibers with the application of various gases during the treatment. The results show enhancement in flexural and tensile strengths and improvement in toughness of the treated samples $[5,10]$.

Previous studies [9, 11 and 12] approve that application of different gases during the plasma treatments may be appropriate for different types of polymeric fibers and have unequal modification effects especially on the Polypropylene [13].

Despite effectiveness of plasma treatment for modification of polymeric surfaces and fibers in concrete application, it still could not be widely used by industries. One reason is the economical consideration, which could be resolved with the development of plasma technology in the close future; another reason of limited applications of plasma in industry is the effect of treatment which may be influenced by factors 
such as temperature and time of application [14]. This effect could be enormously different due to change of the treatment situation and results in unequal productions which is unpleasant for the producers. This problem would also be solved with further development of this technique and reaching more reliable application conditions.

\section{PRACTICAL EXPERIMENTS}

\subsection{Materials and Equipment}

The cement used in all the experiments is CEM I $42.5 \mathrm{R}$ according to EN DIN 197-4. Two different kinds of PCE based superplasticizer, one with hardening acceleration effect (PCE-F) and other with retarding effect (PCE-S) are used to observe the interaction of polymeric based materials in the adhesion enhancement of cement paste to aggregate due to plasma treatment.

In order to ease the observation and interpretation of the results, only one size aggregate is used to make the concrete samples. The aggregate portion passed from sieve \#4mm and stayed on \#2mm (2/4) were washed clean, so that no dirt and dust remained on the surface.

In spite of the variety of plasma treatment types and possibility of different gases during the treatment, and also owing to the limited available research in this field, standard corona discharge with normal pressure air (as the treatment gas) is chosen for this series of assessment due to the availability of device and ease of treatment process for our purpose. This equipment is provided by the Hamburg branch of Tantec business group [15]. The treatment is mainly done with the power of 150 Watt for both aggregate and release agent treatment.

The types of release agent used to evaluate their surface properties are of 3 different kinds:

- Normal mold grease (mineral oil based).

- Ceresit CK 310 (naphthenic mineral oil based); product of Henkel AG \& Co.

- Mikon MG 9006 (polymeric based); product of Münch Chemie International $\mathrm{GmbH}$.

\subsection{Testing and Methods}

Tests were done in the following two main categories.

\subsubsection{Evaluating the Adhesion of Cement Matrix}

\section{and Aggregate due to Plasma Treatment}

Adhesion plays an important role in concrete quality and properties. In concrete, adhesion refers mainly to the bond between the cement matrix and the aggregate surface, known as the interfacial transition zone (ITZ). The ITZ, generally the weakest link of the chain, is considered as the strength-limiting phase in concrete. In addition to the large volume of capillary pores and oriented calcium hydroxide crystals, a major factor responsible for the poor strength of the ITZ in concrete is the presence of micro cracks [16]. The existence of microcracks in the ITZ at the interface with steel and coarse aggregate is the primary reason that concrete is more permeable than the corresponding hydrated cement paste or mortar [16]. In other words, ITZ is partly responsible for the permeability, and also the strength limitation of concrete elements.

However, one possibility to use plasma treatment is to modify the aggregate surface in order to enhance the adhesion properties of it, and consequently influencing the interfacial transition zone. To reach this goal, in this part of the experiment concrete mixes composed of aggregate size 2/4 and cement matrix with and without superplasticizer were produced. The difference of the mixtures is in the treatment of the aggregates, i.e. one group of the aggregates is treated and the other one is not treated.

The assessment is done with examination of the compressive strength and porosity of the samples. The compressive strength tests are performed for the small cubes with the edge dimension of $20 \mathrm{~mm}$ at the age of 1,3,7 and 28 days after casting the sample. As mentioned above, in addition to compressive strength test, the porosity is also examined but only for the samples of 28 days age.

\subsubsection{Evaluating the Interaction of Cement Paste with Release Agent after Plasma Treatment}

The aim of using release agent is to ease the demolding of concrete elements. The mechanism is not complicated: a thin layer of release agent is applied or sprayed on the surface of the mold, which is in contact with the mold surface from one side and would be in contact with concrete from another side after casting the concrete in the molds. The function of the release agent is basically to hinder the cement from sticking to the mold. On the other hand in most of release agents (especially of the hydrophobic types) the low wettability of the release agent makes the cement lime to be more reluctant to be spread out on the surface of the mold, thus the surface takes air bubbles instead of the cement lime and the result is an uneven surface with voids on it.

However, the idea to cover this weakness is to apply plasma treatment technique in order to increase the surface energy and consequently the wettability of the release agent, which is applied on the mold. In other words, the molds could still be removed easily while the final surface is improved in quality and smoothness, due to better spread of the cement lime on the surface of the mold.

In this part, the interaction of fresh cement slurry is evaluated on 3 different types of release agent before and after treatment with plasma. Moreover, the interaction of hardened cement paste is studied on one type of release agent for both treated and untreated cases.

The first test has been performed with tracking a droplet of fresh cement slurry, which is released on the plastic substrate covered with three types of treated and normal release agents. The results are shown in photos and the 
conclusions are made due to the trace of the cement slurry drops and the wettability of those surfaces. In the second test, the normal and treated grease release agent is investigated with inspecting the surface of the hardened cement paste after demolding.

\section{RESULTS AND DISCUSSIONS}

\subsection{Aggregate Plasma Treatment}

This investigation is aimed to increase the bond between the cement matrix and aggregate to improve the interfacial transition zone (ITZ). Owing to the high water-cement ratio, calcium-hydroxide and ettringite crystalline products in the vicinity of the coarse aggregate consist of relatively large crystals, and therefore form a more porous framework than in the bulk cement paste or mortar matrix [16]. Nevertheless, two opposite theories had been argued before the practical tests and observing the results:

First, due to better wettability of aggregate surface after the treatment, the internal bleeding around the aggregates could be increased. Thus, more porous ITZ would be formed and this means a weaker transition zone.

Second, the ITZ would be enhanced owing to better adhesion of the paste to the aggregate surface after plasma treatment.

Because of experimental difficulties in evaluating the bond and ITZ, the assessment was not possible immediately and directly. The quality of bond is generally investigated with measuring the properties of concrete such as compressive and flexural strength, porosity and permeability.

Another challenge of this experiment is the unexplored wettability for the surface of various aggregates. Consequently it is difficult to estimate how long the treatment lasts on aggregate surfaces. Tests in the lab showed that the treatment effects obviously last longer on polymeric surfaces rather than on metallic surfaces. But it was unclear, whether the treatment would affect the aggregate surface and consequently bond to cement matrix or not. On the other hand, in a concrete mixture process the aggregates would be added to the mixer, and the fresh cement lime would be in contact with them during a 5minute long mixing process. This process could result in the treatment effects to be washed away from the treated aggregates.

To overcome these problems, the decision was made to carry out the experiment with one size aggregate (2/4), which is washed thoroughly clean from dirt, dust and fine particles and dried in the oven to constant weight. Then the aggregates were treated 5 minutes as shown in Figure 4 with 150 Watt treatment power and compacted in the prepared cube molds with edge dimension of $20 \mathrm{~mm}$ for each side. Then the cement lime, consisting of cement and water was poured on the aggregates. With vibrating the molds, the cement lime filled all the gaps and space between the aggregates and finally a concrete element with one size aggregate was made. In this way, the cement lime fills the gaps slowly and thus the effects of treatment would not be washed because of mixing process. The cubes were demolded after 24 hours and put under water in $20 \pm 2{ }^{\circ} \mathrm{C}$ for curing until the day of test.
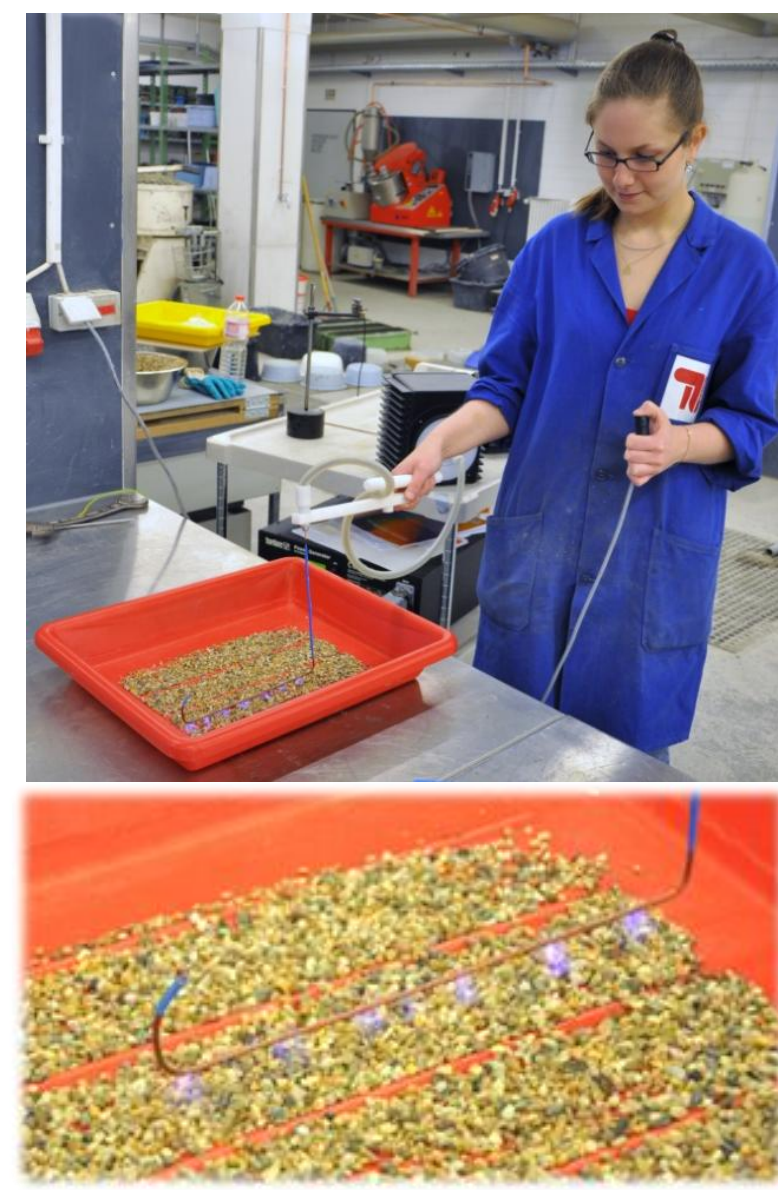

Fig 4: Treatment of prepared aggregate (2/4)

The experiment is done for 6 groups of samples, but only 3 of them presented and discussed in this paper. One of these three mixes is only aggregate and cement paste, while two other mixes had PCE based superplasticizers. Each group includes the control and treated samples. Evaluations are done due to compressive strength of the samples, effective water porosity and absolute porosity which is measured with helium pycnometry. In all the porosity measurements, the effective water porosity is higher than the porosity determined by means of helium pycnometry which complies with the result of previous research works [17]. The contents of the 3 mixtures are presented in Table1.

Table1: Ingredient of different mixtures

\begin{tabular}{|c|c|c|c|c|}
\hline $\begin{array}{l}\text { Ingredien } \\
\mathrm{t}\end{array}$ & $\begin{array}{l}\text { Cemen } \\
t\end{array}$ & $\begin{array}{l}\text { Aggregat } \\
\mathrm{e}\end{array}$ & $\begin{array}{l}\text { Superplasticizer } \\
*\end{array}$ & $\mathrm{w} / \mathrm{c}$ \\
\hline Mix 1 & \multirow{3}{*}{$\begin{array}{l}\text { CEM I } \\
42.5 \mathrm{R}\end{array}$} & \multirow{3}{*}{$2 / 4 \mathrm{~mm}$} & - & $\begin{array}{l}0.4 \\
5\end{array}$ \\
\hline Mix 2 & & & PCE-F & $\begin{array}{l}0.3 \\
5\end{array}$ \\
\hline Mix 3 & & & PCE-S & $\begin{array}{l}0.3 \\
5\end{array}$ \\
\hline
\end{tabular}

* The superplasticizer amount is $1 \%$ of the cement weight. 


\subsubsection{Mix $1(\mathrm{~W} / \mathrm{C}=0.45$, no Superplasticizer $)$}

The compressive strength and porosity diagrams are presented respectively in Chart1 and 2. The results show no considerable difference during and after 28 days both in compressive strength in different ages and also in porosity.

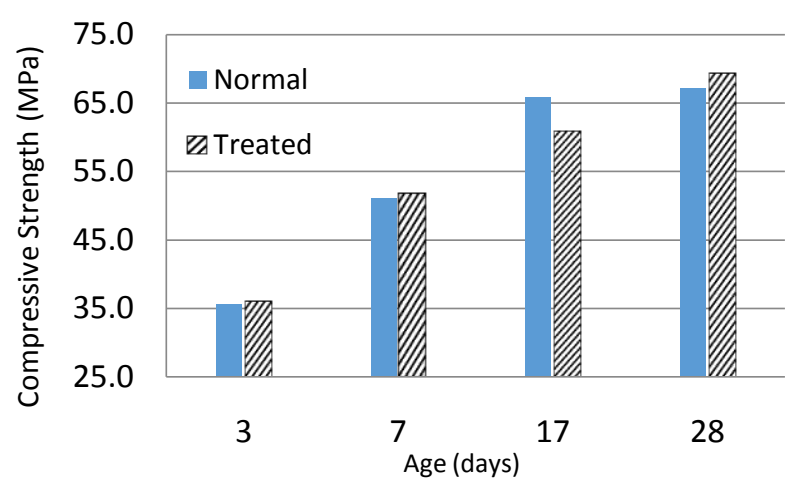

Chart1: Compressive strength diagram of Mix 1 for normal and treated aggregates

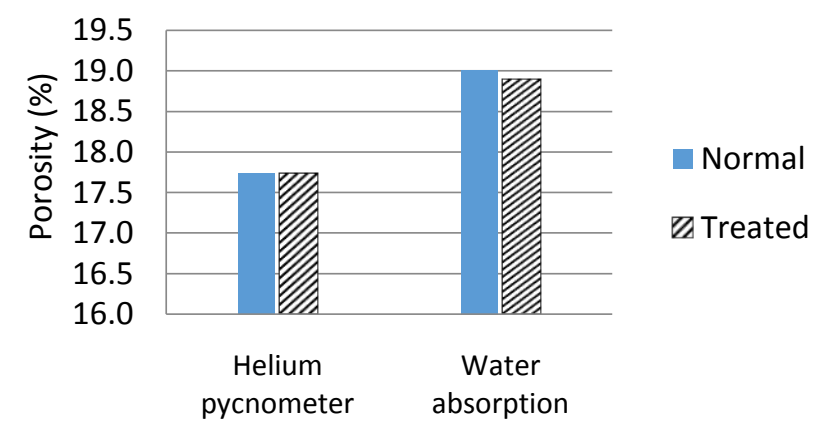

Chart2: Porosity diagram of Mix 1 for normal and treated aggregates at the age of 28 days

\subsubsection{Mix $2(\mathrm{~W} / \mathrm{C}=0.35$, SP. PCE-F 1\%)}

The compressive strength and porosity are presented respectively in Chart 3 and 4 . In these samples the porosity is not considerably different but the strength is nearly up to 5 percent increased.

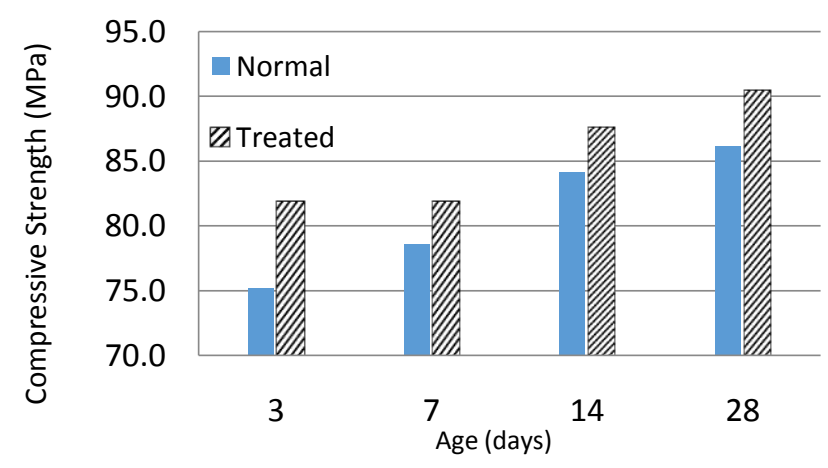

Chart3: Compressive strength of Mix 2 for normal and treated aggregates

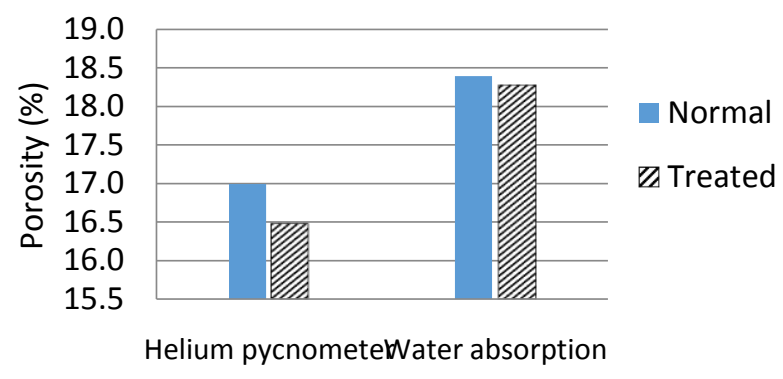

Chart4: Porosity diagram and table of Mix 2 for normal and treated aggregates at the age of 28 days

\subsubsection{Mix $3(\mathrm{~W} / \mathrm{C}=0.35$, Sp. PCE-S 1\%)}

The compressive strength and porosity tables and diagrams are presented in Chart5 and 6. In this group also like the last mix with PCE (Mix 2), the porosity is not considerably affected but the strength is improved again up to 5 percent.

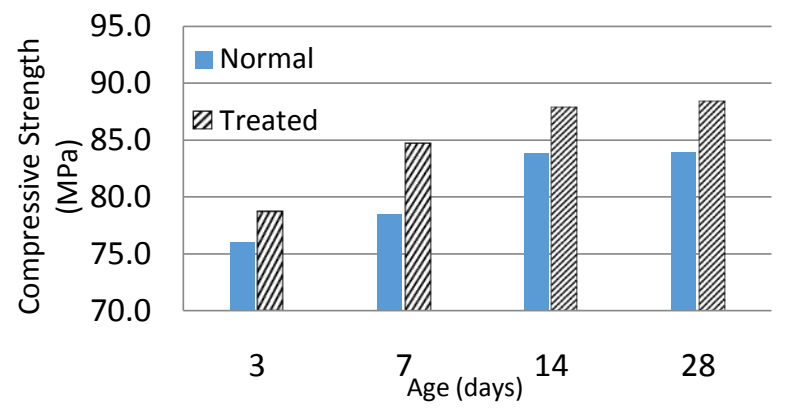

Chart5: Compressive strength of Mix 3 for normal and treated aggregates

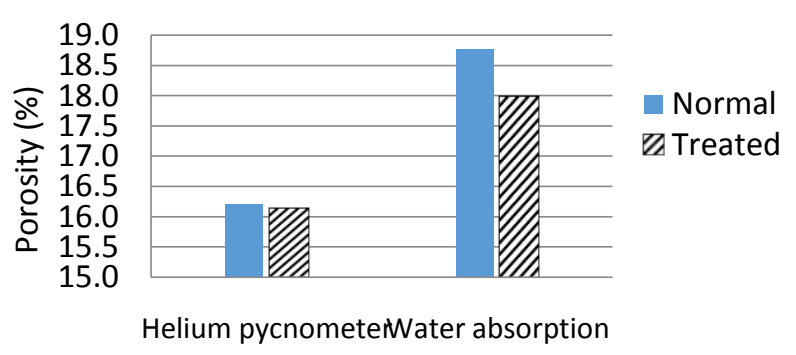

Chart6: Porosity diagram and table of Mix 3 for normal and treated aggregates at the age of 28 days

\subsection{Surface Treatment on Release Agents}

The aim of this study was to observe the effect of plasma treatment on the interaction of cement paste and the release agent on the surface of the mold. In this experiment, different types of release agent are tested with fresh cement slurry and hardened cement paste, with and without plasma treatment.

After applying the release agent on the plastic substrate surface, each surface was treated in two cycles; whereas each cycle means one time surface scan with the plasma treatment. The power of the generator in this treatment was 150 Watt. 
The first observation during the treatment is the smoother and thinner surface of the release agent after the plasma treatment. A pile of release agent is collected on the side of the treated zone (Figure5) and only a very thin layer of release agent remains on the substrate surface.

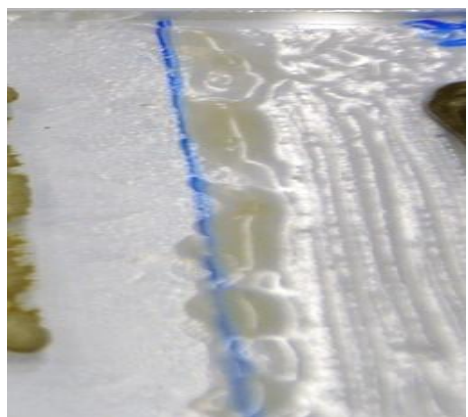

Fig 5: Left side: treated grease; right side: the visible trace of brush with which the grease is applied on the substrate surface; a pile of grease is collected in the middle of the plate due to treatment of the left side

\subsubsection{Fresh Cement Slurry on Release Agent}

As mentioned, three different types of release agent are tested in this part:

1- Grease (mineral oil based);

2- $\quad$ Ceresit CK 310 (naphthenic mineral oil based);

3- Mikon MG 9006 (polymeric based).

These three agents were applied on the surface of 2 plastic substrate plates. One plate is treated with plasma while the other one is kept as normal. The plates were setup in the same angle of 40 degrees to the ground surface. Then a drop the fresh cement paste and a drop of fresh cement slurry were poured on the surface to flow. The comparison and evaluation is done due to the behavior and trace of the cement slurry on the surfaces. The following photos (Figure6-8) show the different behavior of fresh cement slurry on normal (non-treated) and treated release agent.

Figure6 shows the results on grease (mineral oil based) release agent before and after the treatment. It is clear that the cement slurry spreads better on the treated grease surface, because the wettability of the grease is increased due to plasma treatment. This means the finishing surface could be finer as will be illustrated in the next part.

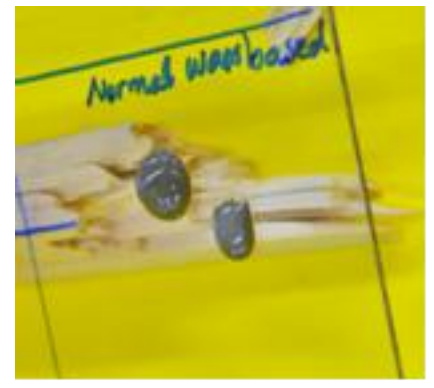

Non-treated surface

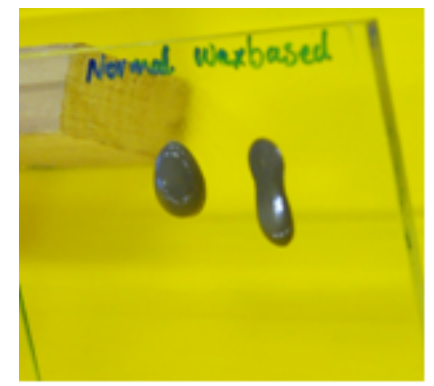

Plasma treated surface
Fig 6: Fresh cement slurry on grease (mineral oil based) release agent
Figure7 shows results of testing on Ceresit CK 310 (mineral oil) release agent. Dissimilar to the interaction of cement slurry with grease release agent (Figure6), the cement slurry spreads out better on the non-treated surface. In other words, the treated surface loses its wettability due to plasma treatment.

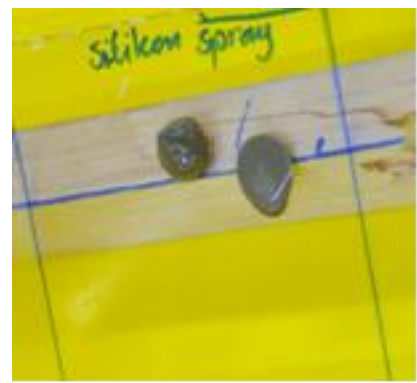

Non-treated surface

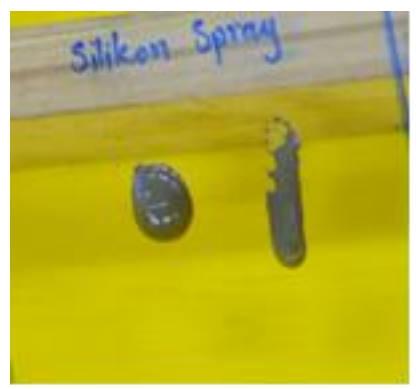

Plasma treated surface
Fig 7: Fresh cement slurry on Ceresit CK 310 (naphthenic mineral oil based) release agent

On the Mikon MG 9006 (polymeric based), the cement slurry spreads out and holds itself on the treated surface (wettability increased), while on the normal surface it slips without any trace to the bottom of the substrate. As could be seen in Figure8, the cement slurry droplet is in the bottom of the non-treated release agent substrate and nothing is left behind.

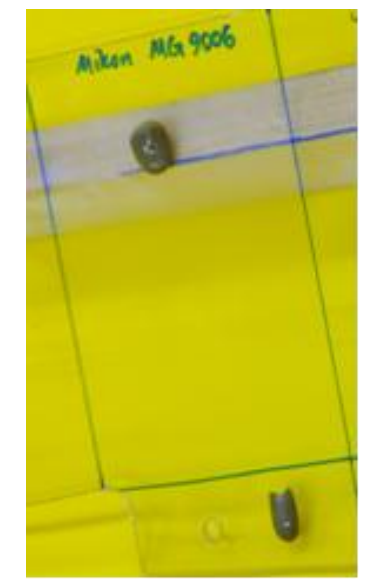

Non-treated surface

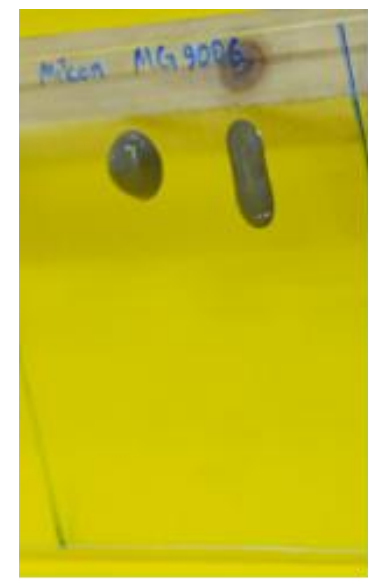

Plasma treated surface
Fig 8: Fresh cement slurry on Mikon MG 9006 (polymeric based) release agent

\subsubsection{Hardened Cement Paste on the Release Agent}

In this part, grease (mineral oil based) release agent is applied on the same substrate in two categories of treated and non-treated. Treatment was done on the release agent with 150 Watt power in two cycles, each cycle with one time surface scanning (Figure9). Then a cylinder PVC mold with $13 \mathrm{~mm}$ thickness is placed on the surface and filled with cement paste (Figure10). After 1 week the samples were removed from the substrate and the surface of the cylinders, which were in contact with release agent, were visually examined. 


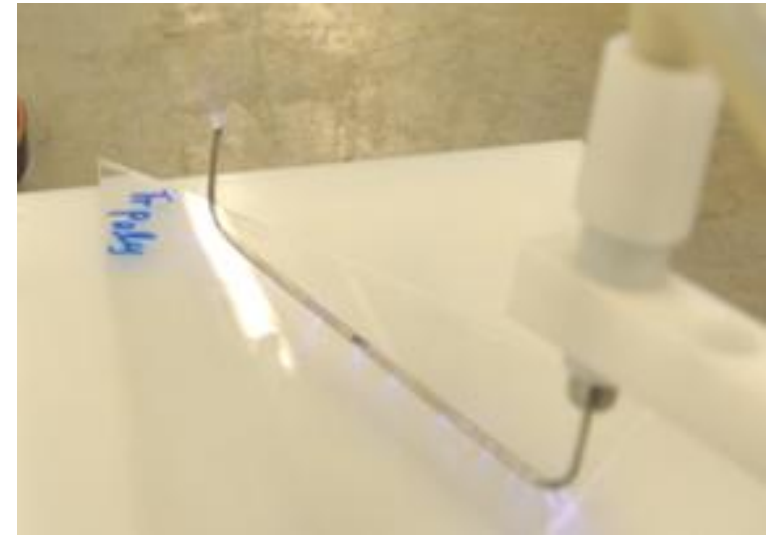

Fig 9: Treatment of a plastic substrate with standard corona discharge

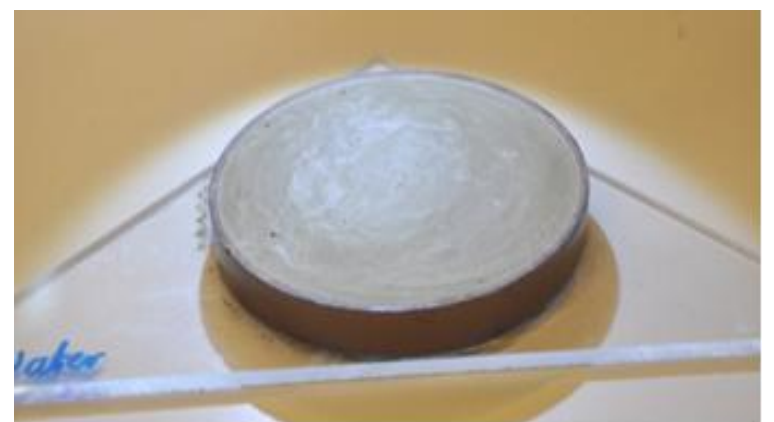

Fig 10: Cylindrical mold on the plastic substrate, filled with cement paste

The first difference, illustrated in Figure11, is the surface roughness of the two samples. The roughness in non-treated sample is higher due to the trace of the brush, with which grease was applied on the mold surface. Another reason for observing a rougher surface on the non-treated sample is the existence of tiny black points (Figure11) on the surface, which happened due to lower wettability of the release agent without the treatment.

Testing the light reflection of the samples (Figure12) showed that the surface of the hardened cement paste on the non-treated grease release agent is shiny like glass, while the surface of the same cement paste on the treated release agent is totally mat.

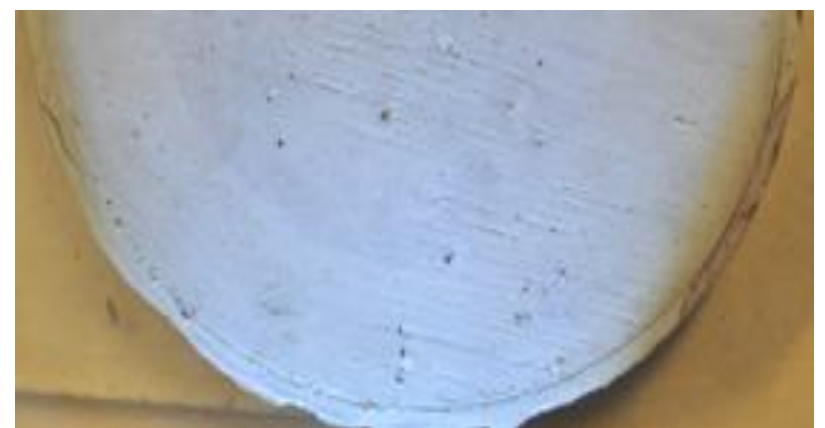

(a) Surface of the hardened cement paste on the non-treated release agent

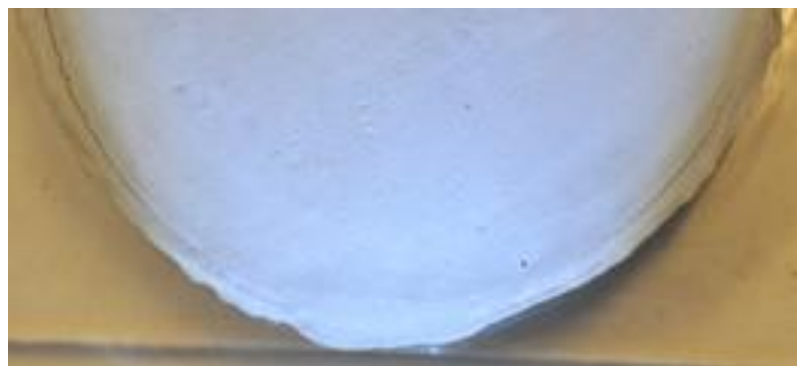

(b) Surface of the hardened cement paste on the treated release agent

Fig 11: Black points in the surface of the sample on nontreated release agent (a) shows a higher roughness comparing to the surface of the sample on treated release agent (b)

\section{CONCLUSIONS}

To sum up, it is clearly observed that the plasma treatment has an affirmative effect on the bond between cement matrix and aggregate surface which was concluded from compressive strength and porosity evaluation. This influence in improving the bond could be far more different applying other types of plasma treatment. However, applying the proper gas such as silica vapor during the treatment could result in a very promising enhancement in interfacial transition zone and in general concrete properties.

Also on the interaction of cement slurry with different release agents, it was observed that not only the physical distribution of release agents on the surface were more uniform, but also the behavior of fresh cement slurry and hardened cement paste are influenced due to standard corona treatment. Different methods with and without gas application for plasma treatment could bring more benefits, but require more experimental works.

\section{ACKNOWLEDGEMENTS}

This research work is done in the Technical Universität Berlin, Department of Building Materials and Construction Chemistry. We would like to acknowledge Tantec $\mathrm{GmbH}$ and especially Mr. Stehnacker for kindly supporting the technical equipment of this work with providing a standard corona generator and its components. We would like also to thank and appreciate worthy endeavor from Dr. R. Herr for all scientific supports. In addition to that, technical support from Berding Beton, which was provided with Mr. A. Munke and his colleagues, is gratefully acknowledged. 


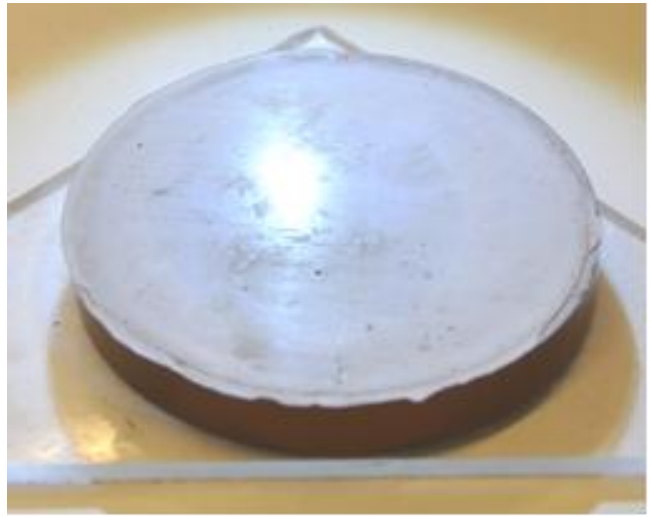

(a) Demolded surface on non-treated grease; shiny reflection

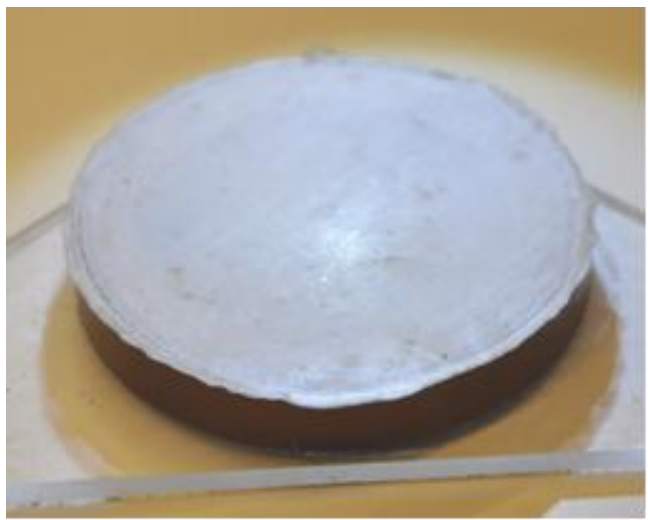

(b) Demolded surface on treated grease; mat reflection

Fig 12: Reflection properties of hardened cement paste on normal (a) and treated (b) grease release agent with the same light condition

\section{REFERENCES}

[1]. A. Fridman, L. A. Kennedy, Plasma Physics and Engineering, Taylor \& Francis, ISBN 1560328487, New York, 2004, pp. 3.

[2]. Luo, Q-Z, D'Angelo, N., Merlino, R.L., Shock formation in a negative ion plasma, J. Physics of Plasma 5 (8) (1998) 2868-2870.

[3]. Gurnett, D.A., Bhattacharjee, A., Introduction to Plasma Physics: With Space and Laboratory Applications, Cambridge, Cambridge University Press, ISBN 0-52136483-3, United Kingdom, 2005, pp. 2.

[4]. "A Unit of Plasma Treatment", Vetaphone.com, (2012). Home - [online] [Accessed 3 May. 2014], Available at: http://www.vetaphone.com/

[5]. Denes, F., Feldman, D., Hua, Z.Q., Zheng, Z., Young, R.A., Cementitious-Matrix Composites from SiCl4-PlasmaActivated Polypropylene Fibres, J. Adhesion Sci. and Tech. 10 (1) (1996) 61-77.

[6]. Liston, E.M., Martinu, L., Wertheimer, M.R., Plasma surface modification of polymers for improved adhesion: a critical review, J. Adhesion Sci. and Tech. 7 (10) (1993) 1091-1127.

[7]. Wheale, S. H., Barker, C.P., Badyal, J.P.S., Chemical Reaction Pathways at the Plasma-Polymer Interface, Langmuir 14 (1998) 6699-6704.
[8]. Bech, L., Lepoittevin, B., El Achhab, A., Lepleux, E., Teule-Gay, L., Boisse-Laporte, C., Roger, P., Double Plasma Treatment-Induced Graft Polymerization of Carbohydrated Monomers on Polyethylene terephthalate Fibers, Langmuir 23 (2007) 10348-10352.

[9]. Zhang, C., Gopalaratnam, V.S., Yasuda, H.K., Plasma Treatment of Polymeric Fibers for Improved Performance in Cement Matrices, J. Applied Polymer Sci. 76 (2000) 19851996.

[10]. Victor C. Li, Hwai-Chung Wu, Yin-Wen Chan, Effect of Plasma Treatment of Polyethylene Fibers on Interface and Cementitious Composite Properties, J. American Ceramic Society 79 (1996) 700-704.

[11]. Feldman, D., Denes, F., Zeng, Z., Denes, A.R., Banu, D., Polypropylene Fiber-Matrix Bonds in Cementitious Composites, J. Adhesion Sci. and Tech. 14 (13) (2000) 1705-1721.

[12]. Zhang, R.F., Li, X.L., Hu, G., Liu, X.S., Yao, Y.G., Study on Surface Structure of Polypropylene Fibers Exposed to Plasma by XPS, J. Functional Polymers (Ch) 7 (1) (1994) 13-17.

[13]. Wang, W., Wang, L., Shi, Q., Yu, H., Chen, T., Wang, C., Sun, T., Progress of the Surface Modification of PP Fiber Used in Concrete, Polymer-Plastics Technology and Engineering 45 (2006) 29-34.

[14]. Shi, Q., Wang, L., Yu, H.Y., Jiang, Sh., The Surface Modification of PP Fiber and its Application in Concrete, Materials Review (Ch) 18 (6) (2004) 58-60.

[15]. Tantec GmbH, Plasma and Corona Treaters, http://www.tantec.com

[16]. Mehta, P. K., Monteiro, P. J. M., Concrete Microstructures, Properties and Materials, McGraw-Hill, ISBN 0-07-146289-9, USA, 2006.

[17]. Marsh et al., B.K., The effect of solvent replacement upon the pore structure characterization of Portland cement paste. Proceedings, Principles and Application of Pore Structural Characterization, Milan, Italy, 1983.

\section{BIOGRAPHIES}

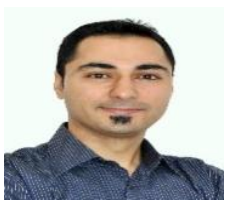

M.Sc. Kasra Shafiei, born 1982 in Iran. Bachelor and Master studies at Technical University of Amir Kabir in Tehran, graduated 2004. Since 2010, PhD student at Technical University of Berlin.

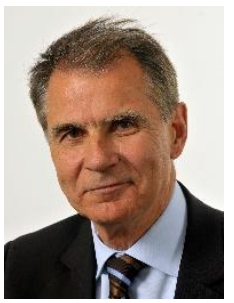

Prof. Dr.-Ing. Bernd Hillemeier, born 1941 in Germany. 1978-1990 the Quality Assurance Manager with the contractor HOCHTIEF. 1990-2009 Uni. Professor in Materials Science at the TU Berlin. 20022008 the chairman of the advisory board of the German Energy Agency. A member of the Executive Boards of acatech (German Academy of Science and Engineering) and BBAW (Berlin Brandenburgische Akademie der Wissenschaften). Since July 2010 elected CEO of the "Friends of the TU Berlin e.V.". 